\title{
Circumstellar Environment around Type la Supernovae of Different Subtypes
}

\author{
Keiichi Maeda* \\ Department of Astronomy, Kyoto University, Kitashirakawa-Oiwake-cho, Sakyo-ku, Kyoto \\ 606-8502, Japan \\ E-mail: keiichi.maeda@kusastro.kyoto-u.ac.jp
}

In this paper, we argue that deriving the nature of circumstellar media (CSM) around type Ia supernovae (SNe Ia) is key to understanding their progenitor system(s). The issue is still controversial for normal SNe Ia, while there is robust evidence of dense CSM for a few classes of SN Ia 'outliers'. We discuss the nature of CSM and the inferred progenitor systems for the candidate super-Chandrasekhar SNe Ia (SC-SNe Ia; SNe Ia originally characterized by large luminosity) and for the so-called SNe Ia-CSM (SNe Ia showing signatures of a strong ejecta-CSM interaction). The nature of CSM around SC-SN Ia 2012dn seems largely consistent with the expectation from an extreme case of the single degenerate scenario. The CSM around SNe Ia-CSM requires a very intensive mass loss rate, which may indicate that an Asymptotic Giant Branch (AGB) star would be involved in their progenitor systems. The link between SN Ia-CSM and SN 1991T-like objects is intriguing, and there is a possibility that they would arise from the same progenitor system while the difference is due to a variance in the progenitor configuration.

The Golden Age of Cataclysmic Variables and Related Objects IV

11-16 September, 2017

Palermo, Italy

\footnotetext{
* Speaker.
} 


\section{Overview on the Outliers of SNe Ia}

Type Ia Supernovae (SNe Ia) represent generally a homogeneous class of stellar explosions. Their light curves follow the so-called Phillips relation (or stretch relation) in a way a brighter object evolves more slowly [1]. Their spectral appearance also forms a one-parameter family, in which their maximum-phase spectra are linked to the brightness at the peak [2]. Within this oneparameter description, the brightest and faintest objects are regarded to be outliers; the bright $\mathrm{SNe}$ Ia are classified as SN 1991T-like SNe Ia [3], while the faint SNe Ia are SN 1991bg-like SNe Ia [4]. There is a tendency that the bright SNe Ia are found in star-forming galaxies while the faint ones prefer passive environment. It has not been clarified if they come from the same progenitor system, or represent multiple progenitor systems.

Recent development in transient surveys and follow-up programs has however casted a doubt on the homogeneous nature of SNe Ia. There is an increasing number of SNe Ia with different features, which does not fit into the above-mentioned one-parameter description. 'Over-luminous' $\mathrm{SNe}$ Ia is a typical example of such outliers $[5,6,7,8$. Originally they were classified by its extremely large luminosity, which exceeds a canonical limit expected by an explosion of a white dwarf (WD) with the Chandrasekhar limiting mass. This is why they are sometimes referred as '(Candidate) Super-Chandrasekhar' SNe Ia (SC-SNe Ia). There are however SNe Ia which show spectral similarity to the over-luminous ones while the peak luminosity is consistent with normal SNe Ia [9 10, and they are also classified as SC-SNe Ia.

In this paper, we focus on normal SNe Ia, SN 1991T-like SNe Ia, and SC-SNe Ia in the following discussion. A comprehensive review on the outliers of SNe Ia, including faint classes, can be found in [11].

\section{Circumstellar Environment around SNe Ia}

Circumstellar (CS) environment is a key to understanding the progenitor(s) of different classes of SNe Ia, as the two most popular progenitor scenarios have totally different predictions. The so-called single degenerate scenario, i.e., a WD that accretes materials from its non-degenerate companion star [12 13], is associated with a dirty CS environment. On the other hand, the double degenerate scenario, i.e., merging binary WDs [14, 15], is generally associated with clean CS environment.

Existence and the amount of CS media (CSM) around 'normal' SNe Ia has been controversial. It has been predicted that the shock-CSM interaction creates non-thermal radio and X-ray emission, which however has not been detected so far. Especially deep limit on the CSM density has been thus obtained for (very) nearby SNe 2011fe and 2014J [16, 17, 18]. On the other hand, a fraction of SNe Ia are found to show time variability in their narrow absorptions created by materials between the SN and the observer [19, 20, 21, 22]. The time variability suggests that the absorption features are originated in the vicinity of the SN so that they are affected by the SN light, therefore it has been suggested as a signature of CSM around SN Ia progenitors. Statistically, even without the time variability, there are more SNe Ia showing the blueshift in the narrow absorption than those showing the redshift [23]. This also indicates that a fraction of SNe Ia are associated with the environment with outflow, naturally expected from the CSM. However, the interpretation requires 
further study. For example, consideration on the ionization of the environment by the SN light suggests that clouds at large distance may be responsible for the formation of the Na ID lines [24], and the blueshift components seen toward SN 2014J are suggested to originate in the interstellar scale [25].
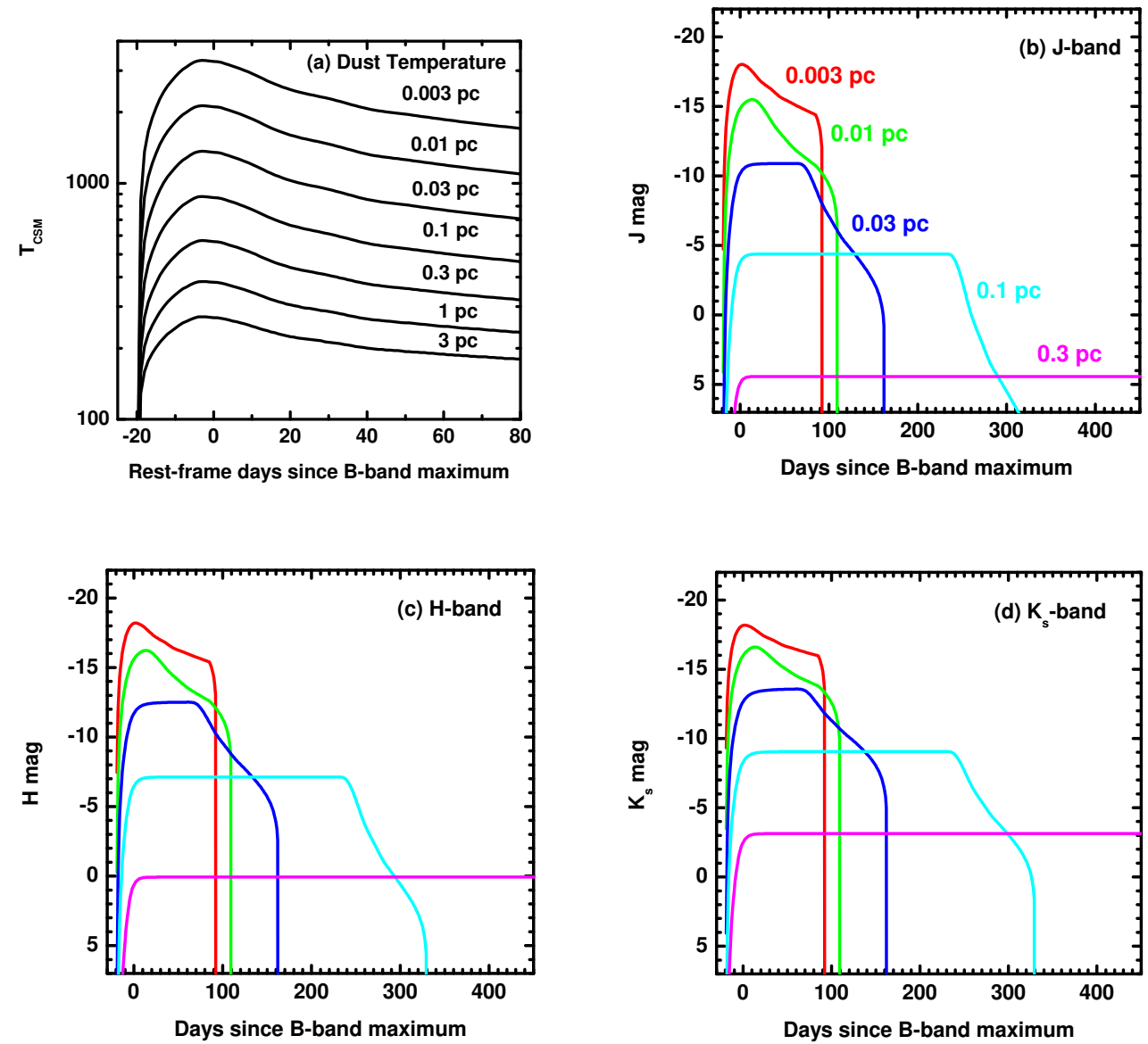

Figure 1: NIR light echo models. (a) Temperature of the CS dust $(T)$ as a function of the rest-frame days, in which the $B$-band maximum is shown for various distances to the CS dust $\left(R=3.24 \times 10^{-3}, 1.02 \times 10^{-2}\right.$, $3.24 \times 10^{-2}, 1.02 \times 10^{-1}, 3.2 \times 10^{-1}, 1.02$, and $3.24 \mathrm{pc}$ from top to bottom). In all of the models, the CS dust mass is set to $10^{-5} M_{\odot}$. (b-d) The echo model light curves for $J$ (b), $H$ (c), and $K_{\mathrm{s}}$-bands (d) as a function of the observed days for the shell model. The color coordinate denotes the model for different distances to the CS dust (red for $R=3.24 \times 10^{-3} \mathrm{pc}$, green for $1.02 \times 10^{-2} \mathrm{pc}$, blue for $3.24 \times 10^{-2} \mathrm{pc}$, cyan for $1.02 \times 10^{-1} \mathrm{pc}$, and magenta for $3.24 \times 10^{-1} \mathrm{pc}$ ) [26]. Reproduced by permission of the Oxford Journals (Fig. 1 of 'Constraining the Amount of Circumstellar Matter and Dust around Type Ia Supernovae through Near-Infrared Echoes’, by K. Maeda, et al., 2015, MNRAS, 452, 3281).

Another potentially strong diagnostics for the existence of CS environment is provided by the expected near-infrared (NIR) echo [26] (Fig. 1). In the single-degenerate scenario, the CS environment is likely polluted not only by gas but also by dust, e.g., as frequently observed for novae or red-giant wind. The SN light will then evaporate the CSM up to $\sim 0.01 \mathrm{pc}$ (for carbon dust) or up to $\sim 0.1 \mathrm{pc}$ (for silicate dust), resulting in the NIR re-emission lasting for a few months 
to an year. Searching for this signal has resulted in null detection for a sample of normal SNe Ia [26].

There are a few classes of SN Ia outliers for which the association with CSM is robustly identified. It is highlighted by the so-called SN Ia-CSM class of objects [27, 28, 29, 30]. They show strong emission lines of hydrogen lines (e.g., $\mathrm{H}_{\alpha}$ ), sharing the spectral appearance with $\mathrm{SNe}$ IIn. The spectral signature announces the on-going and strong hydrodynamical interaction between the SN ejecta and CSM. The difference between the SNe Ia-CSM and SNe IIn is in the property of the underlying SN component. For SNe Ia-CSM, the spectra of SN component are generally matched to $\mathrm{SNe}$ Ia better than to other classes of SNe from massive star progenitors (bet see [31). This is especially evident for PTF $11 \mathrm{kx}$, which shows a clear transition from SN Ia spectra superposed by narrow 'absorption' lines to SN IIn-like spectra characterized by narrow 'emission' lines, at the moment when the SN light curve start deviates from the canonical SN Ia light curve (due to an additional power by the SN-CSM interaction) [32]. Interestingly, the underlying $\mathrm{SNe}$ in $\mathrm{SNe}$ Ia-CSM are generally identified as SN 1991T-like bright SNe Ia [33]. The required mass loss rate for SNe Ia-CSM to power the observed large luminosity is roughly $\sim 10^{-3} M_{\odot} \mathrm{yr}^{-1}$ or even larger, for the mass-loss wind velocity of $\sim 100 \mathrm{~km} \mathrm{~s}^{-1}$ [29].

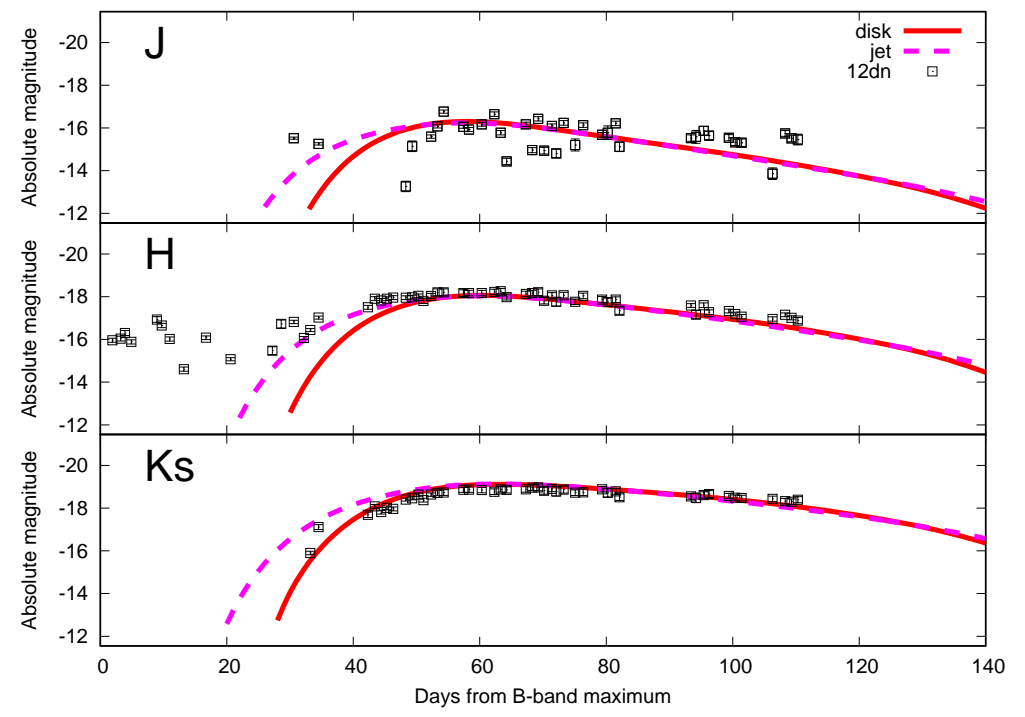

Figure 2: $J, H$, and $K_{s}$-band light curves of the NIE-excess component of SN 2012dn (black-squares). The data points are from [10]. The best fit models are shown for the disk-like CSM configuration (red-solid) and for the bipolar-like configuration (magenta-dashed) [34]. Note that the bipolar configuration is later rejected by [35] based on the consideration of the required density and optical depth, leaving the jet-like configuration as a favored distribution. Reproduced by permission of the AAS (Fig. 1 of 'The Origin of the Near-infrared Excess in SN Ia 2012dn: Circumstellar Dust around the Super-Chandrasekhar Supernova Candidate', by T. Nagao, et al., 2017, ApJ, 835, 143).

Another class of SNe Ia for which the association to dense and massive CSM has been identi- 
fied is the SC-SNe Ia (while the associate so far has been established only for one object) [10]. SN $2012 \mathrm{dn}$ showed a rise in the NIR luminosity at $\sim 40$ days after the maximum brightness. The NIR spectral energy distribution (SED) is well represented by emissions from carbon dust grains near the evaporation temperature. This excessive emission lasted at least for two months with gradual decay in the luminosity and the characteristic temperature. These features are fully consistent with the expectation from the CS echo scenario [26]. The only issue which did not immediately fit into the nature of the predicted NIR echo is the delayed emergence of the NIR excessive emission at $\sim 50$ days after the maximum brightness, as any spherically symmetric shell will lead to the early emergence of the echo component created by dust located toward the direction of the observer [26]. This suggests that the CS dust distribution is asymmetric, without much CS dust toward the direction to the observer. The situation is naturally expected if the CS dust is distributed in a disk-like or jet-like structure, as expected from some mass loss processes from a binary system. It has been shown that the observed NIR SED and light curves can be naturally reproduced by a disk-like CS dust distribution, through radiation transfer simulations (Fig. 2) [34 35]. The required mass-loss rate, under the assumptions of the gas-to-dust ratio of 100 and the mass-low wind velocity of 10 $\mathrm{km} \mathrm{s}^{-1}$, is $1.6 \times 10^{-5} M_{\odot} \mathrm{yr}^{-1}$.

\section{Further Implications}

The progenitor system of SNe Ia-CSM should experience the exceedingly large mass loss rate just before the explosion. They show the signature of the interaction at least for a few months. Assuming the mass-loss wind velocity of $\sim 100 \mathrm{~km} \mathrm{~s}^{-1}$, this translates to the mass loss history in the last few decades (which is proportional to the assumed mass loss velocity), and the mass of CSM exceeding $\sim 0.01 M_{\odot}$ within $\sim 10^{16} \mathrm{~cm}$. This may involve an Asymptotic Giant-branch (AGB) star. For example, the so-called 'type 1.5' $\mathrm{SN}$ [36, a thermonuclear runaway of the $\mathrm{CO}$ core within a massive AGB, is a possible candidate [27]. Another possibility would be the coredegenerate scenario, i.e., a merger of a WD and an AGB [37,38].

An interesting indication is that the underlying SNe in SNe Ia-CSM are generally of the SN $1991 \mathrm{~T}$ class. It would be possible that SNe 1991T-like SNe Ia and SNe Ia-CSM share essentially the same origin and progenitor system, while the difference could be created by a variance within the same scenario, i.e., a similar amount of CSM located in the vicinity of the SN progenitor ( $\mathrm{SNe}$ Ia-CSM) or at a distance (SNe 1991T-like). In this view, the Kepler SN remnant, which has been argued to be an outcome of an SN 1991T-like SN Ia, may provide a hint [39]. From the X-ray data, [39] derived that it had a cavity in the CSM distribution, surrounded by a massive CSM $\left(\sim 0.3 M_{\odot}\right.$ or larger) at $\sim 2 \mathrm{pc}$ from the progenitor system. This suggests that the CSM was hit at $\sim 200$ years and would have been classified as an SN 1991T-like object, not as an SN IA-CSM, if it would have been spectroscopically observed in the SN phase. The average mass loss rate would have been $>10^{-5} M_{\odot} \mathrm{yr}^{-1}$ in the last $10^{4}$ years, which might reach to the level similar to SN Ia-CSM if it would have been ejected in a time scale of $\sim 100$ years.

As for SC-SN 2012dn, the derived mass loss rate may be consistent with an extreme case of a symbiotic system within the single degenerate scenario. The disk-like geometry may also be consistent with the scenario, if the mass loss is confined in the orbital plane. It should however be noted that the similar NIR echo was not observed for another well-studied SC-SN Ia 2009dc, with 
the upper limit in the CSM density smaller than the CSM density around SN 2012dn by an order of magnitude [34]. This might indicate that SC-SNe Ia may be further divided into two classes. Indeed, SN $2009 \mathrm{dc}$ is characterized by a large luminosity and slow expansion velocity as originally attributed to this class of objects, while SN 2012dn is characterized by a relatively low luminosity and large expansion velocity while the overall spectral characteristics is shared by the two.

Given the derived disk-like geometry of CSM around SN 2012dn, it is expected that further insight can be obtained through polarimetric observations. Indeed, the CSM as derived for SN $2012 \mathrm{dn}$ should create a strong polarization signal, up to $\sim 8 \%$ depending on the viewing direction [35]. The polarization observation, still rare especially for SN Ia outliers, is therefore strongly encouraged to reveal the nature of CSM around SN Ia progenitors.

\section{Summary}

In this paper, we have argued that deriving the existence/nature of CSM around SNe Ia is key in understanding the progenitor system(s). The issue is still controversial for normal SNe Ia. We present evidence of dense CSM around classes of SN Ia 'outliers', focusing on the so-called SNe Ia-CSM and SC-SNe Ia.

The nature of CSM for SC-SN Ia 2012dn seems largely consistent with the expectation from an extreme case of the single degenerate path. This is so far only one example among SC-SNe Ia (with another upper limit for SN 2009dc) where the deep NIR observation allows a meaningful constraint on the nature of CSM. Follow-up observations both in NIR photometry/spectroscopy and optical polarization is highly encouraged for future SC-SNe Ia.

The CSM around SNe Ia-CSM requires a very intensive mass loss rate, which may indicate that an AGB would be involved in their progenitor systems. The link to SN 1991T-like objects is intriguing. A long-term follow-up of SN 1991T-like SNe Ia could provide an interesting possibility, which may pick up some 'SN 1991T-like' objects turned into 'SNe Ia-CSM', similar to the case we observe for core-collapse $\mathrm{SNe}$ showing transition to $\mathrm{SNe}$ IIn due to a delayed emergence of the SN ejecta-CSM interaction 440,41].

\section{References}

[1] Phillips, M.M., et al. 2013, ApJ, 779, 38

[2] Nugent, P., Phillips, M.M., Baron, E., Branch, D., Hauschildt, P. 1995, ApJ, 455, L147

[3] Filippenko, A.V., et al. 1992a, ApJ, 384, L15

[4] Filippenko, A.V. et al. 1992b, AJ, 3104, 1543

[5] Howell, D.A., et al. 2006, Nature, 443, 308

[6] Hicken, M. et al. 2007, ApJ, 669, L17

[7] Yamanaka, M., et al. 2009, ApJ, 707, L118

[8] Scalzo, R.A., et al. 2010, ApJ, 713, 1073

[9] Chakradhari, N.K. et al. 2014, MNRAS, 443, 1663

[10] Yamanaka, M., et al. 2016, PASJ, 68, 68 
[11] Maeda, K., Terada, Y. 2016, International Journal of Modern Physics D, 25, Issue 10, 1630024

[12] Whelan, J., Iben, I. Jr. 1973, ApJ, 186, 1007

[13] Nomoto, K. 1982, ApJ, 253, 798

[14] Iben, I. Jr., Tutukov, A.V. 1984, ApJ, 284, 719

[15] Webbink, R.F. 1984, ApJ, 277, 355

[16] Margutti, R., et al. 2012, ApJ, 751, 134

[17] Margutti, R., et al. 2014, ApJ, 790, 52

[18] Pérez-Torres, M.A., et al. 2014, ApJ, 792, 38

[19] Patat, F., et al. 2007, Science, 317, 924

[20] Simon, J.D., et al. 2009, ApJ, 702, 1157

[21] Blondin, S., et al. 2009, ApJ, 693, 207

[22] Sternberg, A., et al. 2014, MNRAS, 443, 1849

[23] Sternberg, A., et al. 2011, Science, 333, 856

[24] Chugai, N. N. 2008, AstL, 34, 389

[25] Maeda, K., et al. 2016, ApJ, 816, 57

[26] Maeda, K., Nozawa, T., Nagao, T., Motohara, K. 2015a, MNRAS, 452, 3281

[27] Hamuy, M., et al. 2003, Nature, 424, 651

[28] Aldering, G., et al. 2006, ApJ, 650, 510

[29] Tadia, F., et al. 2012, A\&A, 545, L7

[30] Silverman, J.M., et al. 2013, ApJS, 207, 3

[31] Benetti, S., et al. 2006, ApJ, 653, L129.

[32] Dilday, B., et al. 2012, Science, 337, 942

[33] Leloudas, G., et al. 2015, A\&A, 574, 61

[34] Nagao, T., Maeda, K., Yamanaka, M. 2017, ApJ, 835, 143

[35] Nagao, T., Maeda, K., Yamanaka, M. 2018, ApJ, in press (arXiv:1802.08954)

[36] Iben, I. Jr., Renzini, A. 1983, ARAA, 21, 271

[37] Sparks, W.M., Stecher, T.P., 1974, ApJ, 188, 149

[38] Soker, N., 2015, MNRAS, 450, 1333

[39] Katsuda, S., et al. 2015, ApJ, 808,49

[40] Maeda, K., et al. 2015b, ApJ, 807, 35

[41] Kuncarayakti, H., et al. 2018, ApJ, 854, L14 